\title{
Method for Estimating the Parameters of LFM Radar Signal
}

\author{
Chuan-Zhang TAN ${ }^{1, a}$, Hong-Wei $\mathrm{LI}^{1}$ and Chang-Zhou FAN ${ }^{1}$ \\ ${ }^{1}$ School of Information and Navigation, Air Force Engineering University, Xi'an 710077, China.
}

\begin{abstract}
In order to obtain reliable estimate of parameters, it is very important to protect the integrality of linear frequency modulation (LFM) signal. Therefore, in the practical LFM radar signal processing, the length of data frame is often greater than the pulse width (PW) of signal. In this condition, estimating the parameters by fractional Fourier transform (FrFT) will cause the signal to noise ratio (SNR) decrease. Aiming at this problem, we multiply the data frame by a Gaussian window to improve the SNR. Besides, for a further improvement of parameters estimation precision, a novel algorithm is derived via Lagrange interpolation polynomial, and we enhance the algorithm by a logarithmic transformation. Simulation results demonstrate that the derived algorithm significantly reduces the estimation errors of chirp-rate and initial frequency.
\end{abstract}

\section{Introduction}

As a non-stationary signal, LFM signal is widely applied to the fields of radar, sonar and communication. Scholars have done a lot of research on the parameters estimation of LFM signal. Among the numerous algorithms have presented, the FrFT is very suitable for LFM signal detection and parameters estimation because it can be interpreted as a decomposition of orthogonal LFM functions. FrFR was first proposed by Namias in 1980 [1]. It was not widely used until 1996 that Ozaktas presented the digital fractional Fourier transform (DFrFT) [2], which could be fast implemented by fast Fourier transform (FFT). However, the resolution of DFrFT is fixed, in order to solve this problem, a high resolution FrFT algorithm which named Zoom-FrFT and single point FrFT algorithm named SP-FrFT are proposed in [3], as an application, a new algorithm combing ZoomFrFT with short-time Fourier transform (STFT) is presented to estimate the parameters of LFM signal [4]. Another method is using curve fitting over the spectral line via moving least square (MLS) [5], but it will produce an additional computation. The direct interpolation algorithm [6] can obtain high accuracy of parameters estimation while doesn't increase the computational complexity, and the iterative interpolation algorithm [7] further reduced the estimation error, but they are effective only to the no windowing signal. However, in fact, the windowed LFM signal also has to be considered [8, 9]. The Gaussian-weighted fractional Fourier transform (GFrFT) [10] is presented for the parameters estimation of low duty ratio signal, and it reduces estimation errors combining with fourth-order algorithm (F-GFrFT) [11], but the research found its performance can be further improved.

On the basis of analyzing the problem occurs in practical radar signal processing, a new method for estimating the parameters of LFM signal is proposed in this paper. Firstly, the basic principle of

\footnotetext{
${ }^{a}$ Corresponding author: kdytcz@163.com
} 
parameters estimation based on FrFT is given. And then, aiming at the reality that the length of data frame is greater than the PW of signal, we establishe a new model for parameters estimation of low duty ratio signal, and windowed the data frame with a Gaussian window to improve the SNR. In order to realize the windowed LFM signal parameters estimation, a novel algorithm is derived from Lagrange interpolation polynomial, and its performance improved via logarithmic scale transform. We also compare the performance of the proposed algorithm with F-GFrFT and Cramer- Rao lower bound (CRLB) [12], simulation results demonstrate the validity and superiority of the proposed estimator.

\section{Parameters estimation of LFM signal based on FrFT}

Let $x(t)$ denote a continuous LFM signal, the $p$ th-order fractional Fourier transform $X_{\alpha}(u)$ of $x(t)$ could be defined as

$$
X_{\alpha}(u)=F^{p}[x(t)]=\int_{-\infty}^{\infty} x(t) K_{\alpha}(u, t) \mathrm{d} t
$$

where $\alpha=p \pi / 2, p$ is an arbitrary real number, $F^{p}[\cdot]$ denotes the operator notation of FrFT. $K_{\alpha}(u, t)$ is the kernel function of FrFT, and its definition is given by

$$
K_{\alpha}(u, t)= \begin{cases}A_{\alpha} \exp \left\{j \pi\left[\cot \left(u^{2}+t^{2}\right)-2 u t \csc \alpha\right]\right\}, \quad \alpha \neq K \pi \\ \delta(t-u), \quad \alpha=2 K \pi \\ \delta(t-u), \quad \alpha=(2 K+1) \pi\end{cases}
$$

where $A_{\alpha}=\sqrt{1-j \cot \alpha}, K$ is an integer.

It demands the implementation of discrete FrFT on a digital signal processor (DSP) in practical application, so we should get the digital computation of the FrFT. It is desirable that Ozaktas proposed a decomposition algorithm which is called DFrFT, it is widely used because of its low computation complexity. The model of a discrete LFM signal with an additive white Gaussian noise (with zeromean and variance $\sigma^{2}$ ) is given by

$$
x(n)=s(n)+w(n)=b \exp \left(j \theta+\frac{j 2 \pi f n}{f_{s}}+\frac{j \pi f n^{2}}{f_{s}^{2}}\right)+w(n), n \in\left[-\frac{N-1}{2}, \frac{N-1}{2}\right]
$$

where $N$ is the length of LFM signal, $n$ is an integer, $b$ is the amplitude, $\theta$ is the initial phase, $f$ is the initial frequency, $f_{s}$ is the sampling frequency and $k$ denotes the chirp-rate. When the $\alpha$ rotates counterclockwise to the optimal order, the energy of LFM signal will be best gathered in the twodimensional plane, and $\left|X_{\alpha_{0}}\left(u_{0}\right)\right|^{2}$ reaches its peak value at $\left(\alpha_{0}, u_{0}\right)$. Therefore, the chirp-rate and initial frequency can be estimated by

$$
\left\{\begin{array}{l}
\hat{k}=-\cot \alpha_{0} \frac{f_{s}^{2}}{N_{N}} \\
\hat{f}=u_{0} \csc \alpha_{0} \frac{f_{s}}{N}
\end{array}\right.
$$

The estimation error comes from two aspects: (1) restriction of search step size in $\alpha$ domain, the estimation accuracy can be improved by reducing the size of search step while the computation cost is increased by series; (2) the fence effect caused by discretization in $u$ domain. To improve the estimation accuracy and ensure the computation cost not significantly increased, the interpolation method is a splendid way. 


\section{Model for parameters estimation of low duty ratio signal}

The chirp-rate and initial frequency of LFM signal are the most important measurements for radar signal sorting and identification. In practical signal processing, the length of data frame is much larger than the PW of LFM signal. We can get a coarse estimation of PW by envelope detection etc. to reduce the length of processing data, but it is still longer than the LFM signal. To avoid the SNR decrease by estimating the parameters directly, we can multiply the processing data with a Gaussian window function. Therefore, it becomes a problem of parameters estimation under low duty ratio. The diagram of processing model is as shown in Figure 1.

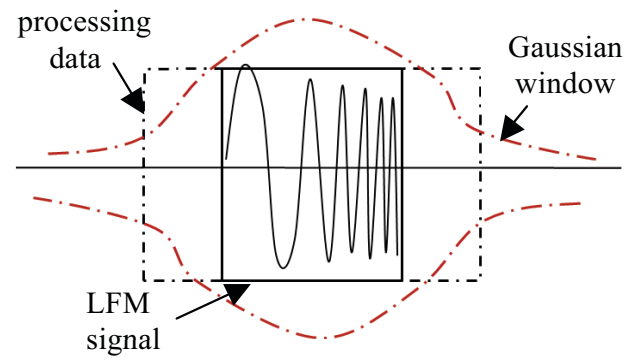

Figure 1. The diagram of signal processing model

According to Eq. (3), the expression of the signal processing model can be written as

$$
x(n)=[s(n)+w(n)] g(n), n \in\left[-\frac{L-1}{2}, \frac{L-1}{2}\right]
$$

where

$$
\left\{\begin{array}{l}
s(n)=b \exp \left(j \theta+\frac{j 2 \pi f n}{f_{s}}+\frac{j \pi k n^{2}}{f_{s}^{2}}\right) \operatorname{rect}\left(\frac{n}{L}\right) \\
g(n)=\exp \left(-\frac{2 c^{2}}{L^{2}} n\right)
\end{array}\right.
$$

where $\mathrm{g}(\mathrm{n})$ is a Gaussian function, $\mathrm{c}$ is an integer which takes control of the shape of the Gaussian window, $L$ denotes the length of processing data. The ${ }^{\operatorname{rect}\left(\frac{n}{L}\right)}$ is given by

$$
\operatorname{rect}\left(\frac{n}{L}\right)=\left\{\begin{array}{l}
1, n \in\left[-\frac{N-1}{2}, \frac{N-1}{2}\right] \\
0, n \in\left[-\frac{L-1}{2},-\frac{N-1}{2}\right) \cup\left(\frac{N-1}{2}, \frac{L-1}{2}\right]
\end{array}\right.
$$

So the duty ratio is defined as

$$
d=\frac{N}{L}
$$

The output SNR of FrFT [13] is defined as 


$$
\mathrm{SNR}_{\text {out }}=\frac{N^{2} \mathrm{SNR}_{\text {in }}^{2}}{\frac{2 N \mathrm{SNR}_{\text {in }}}{d}+\left(\frac{1}{d}\right)^{2}}
$$

where $\mathrm{SNR}_{\text {in }}=\frac{b^{2}}{\sigma^{2}}$, and the output SNR of the new signal processing model can be defined as [10]

$$
\mathrm{GSNR}_{\text {out }}=\frac{N^{2} \mathrm{SNR}_{\text {in }}^{2}}{2 N \operatorname{SNR}_{\text {in }}\left(\frac{\eta_{2} \rho d}{\eta_{1}^{2}}\right)+\left(\frac{\eta_{2} \rho d}{\eta_{1}^{2}}\right)^{2}}
$$

where

$$
\left\{\begin{array}{l}
\eta_{1}=\operatorname{erf}\left(\frac{c}{\sqrt{2}} d\right) \\
\eta_{2}=\operatorname{erf}(c) \\
\rho=\frac{c}{\sqrt{\pi}}
\end{array}\right.
$$

$\operatorname{erf}(\cdot)$ is error function.

The same model is used in [10] and GFrFT algorithm is presented to estimate the parameters of LFM signal, but GFrFT only improves the SNR, although it improves the estimation accuracy combining with fourth-order algorithm, there still is a large gap compared with CRLB. In this context, we need to seek an algorithm with higher estimation accuracy.

\section{Quadratic interpolation algorithm based on FrFT}

The conventional interpolation algorithms are derived from properties of the rectangular window (no window), it is not suitable for the parameters estimation of windowed signal. The essence of the quadratic interpolation derived from Lagrange interpolation polynomial is parabolic fitting, so it can be applied to arbitrary windowed signal.

The Lagrange polynomial is given by

$$
\left\{\begin{array}{l}
\chi=l_{i-1} \chi_{i-1}+l_{i} \chi_{i}+l_{i+1} \chi_{i+1} \\
l_{i-1}=\frac{\left(\kappa-\kappa_{i}\right)\left(\kappa-\kappa_{i+1}\right)}{\left(\kappa_{i-1}-\kappa_{i}\right)\left(\kappa_{i-1}-\kappa_{i+1}\right)} \\
l_{i}=\frac{\left(\kappa-\kappa_{i-1}\right)\left(\kappa-\kappa_{i+1}\right)}{\left(\kappa_{i}-\kappa_{i-1}\right)\left(\kappa_{i}-\kappa_{i+1}\right)} \\
l_{i+1}=\frac{\left(\kappa-\kappa_{i-1}\right)\left(\kappa-\kappa_{i}\right)}{\left(\kappa_{i+1}-\kappa_{i-1}\right)\left(\kappa_{i+1}-\kappa_{i}\right)}
\end{array}\right.
$$

we can get a function via Eq. (12) and the graph of the function is a parabola, we can obtain the estimated value of the target parameter by calculating the vertex coordinates, the expression of the estimator is given by

$$
\hat{\kappa}=\kappa_{\mathrm{i}}+\frac{1}{2} \frac{\chi_{i-1}-\chi_{i+1}}{\chi_{i-1}-2 \chi_{i}+\chi_{i+1}}
$$


By searching the spectrum peak in the two-dimensional plane, we can get the peak coordinates in $\alpha$ domain $\left(\kappa_{p_{i}}, \chi_{p_{i}}\right)$ and in $u$ domain $\left(\kappa_{u_{i}}, \chi_{u_{i}}\right)$, and $\chi_{p_{i}}=\chi_{u_{i}}$. In $\alpha$ domain, the estimator can be obtained by substituting the coordinate of vertex and its adjacent points into the Eq. (13)

$$
\hat{\kappa}_{p}=\kappa_{p_{i}}+\frac{1}{2} \frac{\chi_{p_{i-1}}-\chi_{p_{i+1}}}{\chi_{p_{i-1}}-2 \chi_{p_{i}}+\chi_{p_{i+1}}}
$$

But in $u$ domain, the width of main lobe is less than three bins [14], we have to calculate the coordinates of other two adjacent points $\left(\kappa_{u_{i-0.5}}, \chi_{u_{i-0.5}}\right)$ and $\left(\kappa_{u_{i+0.5}}, \chi_{u_{i+0.5}}\right)$ to the vertex by SP-FrFT algorithm. The expression of estimator is given in three cases

Case $1 \chi_{u_{i-0.5}}<\chi_{u_{i}}<\chi_{u_{i+0.5}}$ or $\chi_{u_{i+0.5}}<\chi_{u_{i}}<\chi_{u_{i-0.5}}$

$$
\hat{\kappa}_{u}=\kappa_{u_{i}}+\frac{1}{4} \frac{\chi_{u_{i-0.5}}-\chi_{u_{i+0.5}}}{\chi_{u_{i-0.5}}-2 \chi_{u_{i}}+\chi_{u_{i+0.5}}}
$$

Case $2 \chi_{u_{i}}<\chi_{u_{i+0.5}}<\chi_{u_{i+1}}$

$$
\hat{\kappa}_{u}=\kappa_{u_{i}}+\frac{1}{4} \frac{3 \chi_{u_{i}}-4 \chi_{u_{i+0.5}}+\chi_{u_{i+1}}}{\chi_{u_{i}}-2 \chi_{u_{i+0.5}}+\chi_{u_{i+1}}}
$$

Case $3 \chi_{u_{i-1}}<\chi_{u_{i-0.5}}<\chi_{u_{i}}$

$$
\hat{\kappa}_{u}=\kappa_{u_{i}}-\frac{1}{4} \frac{\chi_{u_{i-1}}-4 \chi_{u_{i-0.5}}+3 \chi_{u_{i}}}{\chi_{u_{i-1}}-2 \chi_{u_{i-0.5}}+\chi_{u_{i}}}
$$

Considering that the main lobe of Gaussian windowed signal is also Gaussian-shaped, we can use logarithmic scaling transform to the magnitude of the FrFT to improve the precision of parabola fitting. As an enhancement algorithm, its universal expression is the rewriting of Eq. (13)

$$
\hat{\kappa}=\kappa_{\mathrm{i}}+\frac{1}{2} \frac{\mathrm{f}\left(\chi_{i-1}\right)-\mathrm{f}\left(\chi_{i+1}\right)}{\mathrm{f}\left(\chi_{i-1}\right)-2 \mathrm{f}\left(\chi_{i}\right)+\mathrm{f}\left(\chi_{i+1}\right)}
$$

where $\mathrm{f}(\cdot)$ is the operator notation of natural logarithm, we can obtain the different expression form in $u$ domain too, and the Eq. (18) is valid because $\mathrm{f}(\cdot)$ is monotonic. Both the quadratic interpolation algorithm and its enhanced version are simulated.

\section{Simulations and results analysis}

\subsection{The SNR gain with different $c, d$ and $S N R_{\text {in }}$}

In order to prove that the new signal processing model can improve the SNR, we defined $R$ as

$$
R=\frac{\mathrm{GSNR}_{\text {out }}}{\mathrm{SNR}_{\text {out }}}
$$


Our destination is to get the optimal $c$ with different values of $d$ and $\mathrm{SNR}_{\text {in }}$, but $R$ is a function of $c, d$ and $\mathrm{SNR}_{\text {in }}$. Firstly, we can get the relationship between $R$ and $d, c$ while $\mathrm{SNR}_{\text {in }}$ is a constant (as shown in Figure 2), and then analysis the relationship between $R$ and $\mathrm{SNR}_{\text {in }}$ while $d$ and $c$ are constants (as shown in Figure 3).

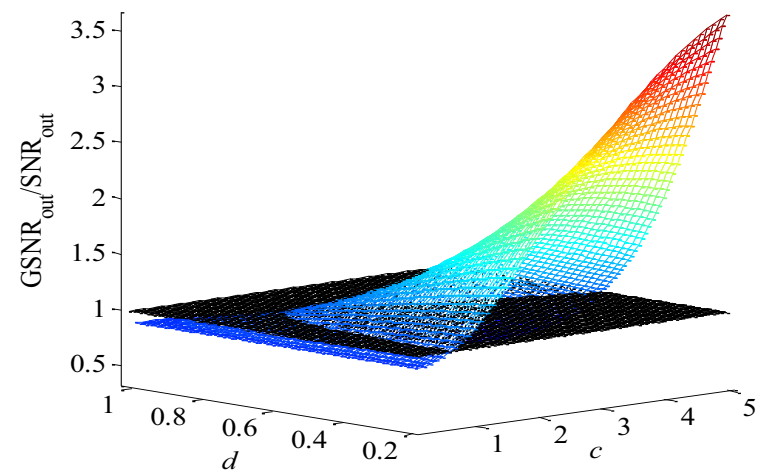

Figure 2. The relationship between $R$ and $d, c$

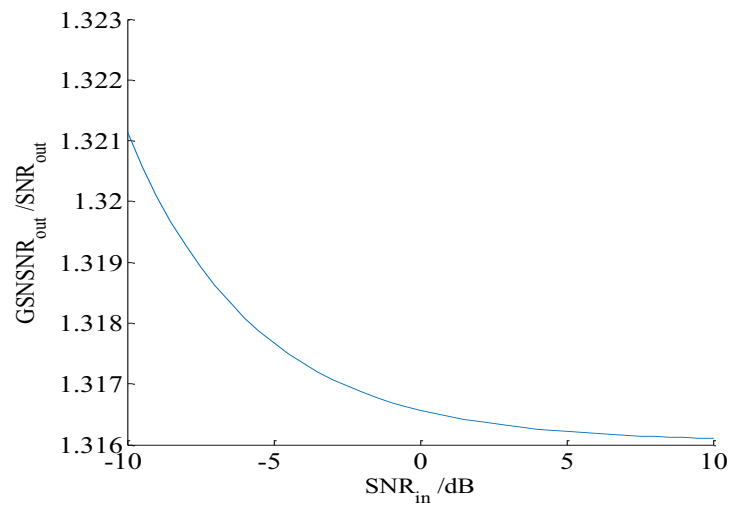

Figure 3. The relationship between $R$ and $\mathrm{SNR}_{\text {in }}$

From the Figure $2\left(\mathrm{SNR}_{\text {in }}=1\right)$, we can see that the new signal processing model could improve the SNR under low duty ratio, and the lower the duty ratio is, the more the SNR improved. From the figure $3(d=0.6, c=2.31)$, we can get that the lower the $\mathrm{SNR}_{\text {in }}$ is the more the SNR improved. In summary, the new signal model is especially suitable for the estimation of LFM signal under low duty ratio and low SNR environment. In order to obtain the optimal $c$ for different $d$, we take $\mathrm{SNR}_{\text {in }}=1$ as an example and get the following table 
Table 1.Optimal $c$ for different $d\left(\mathrm{SNR}_{\text {in }}=1\right)$

\begin{tabular}{ccc}
\hline Duty ratio $(d)$ & Optimal $c$ & $\mathrm{GSNR}_{\text {out }} / \mathrm{SNR}_{\text {out }}(R)$ \\
\hline 0.2 & 5.00 & 3.6670 \\
0.3 & 4.67 & 2.6321 \\
0.4 & 3.50 & 1.9733 \\
0.5 & 2.80 & 1.5783 \\
\hline 0.6 & 2.31 & 1.3163 \\
0.7 & 1.93 & 1.1331 \\
0.8 & 1.56 & 1.0077 \\
0.9 & 1.13 & 1.0044 \\
1.0 & $0.01(0)$ & $0.9203(1)$ \\
\hline
\end{tabular}

When the $d=1$, we can take $c=0$, and then the Gaussian window changes into a rectangular window.

\subsection{The performance of estimator for different $S N R_{\text {in }}$}

We take the latest algorithm F-GFrFT which combines the advantage of GFrFT and fourth-order algorithm as a contrast algorithm, and set $N=513, L=1023, b=1, \theta=\pi / 4, f=24.3 \mathrm{MHz}, f_{s}=100 \mathrm{MHz}$, $k=4.76 \mathrm{MHz} / \mathrm{us}, \mathrm{c}=2.8$, and run 100 Monte Carlo simulations, and then calculate the normalized root mean square error (NRMSE) of chirp-rate and initial frequency. The CRLB is also simulated within the figure.

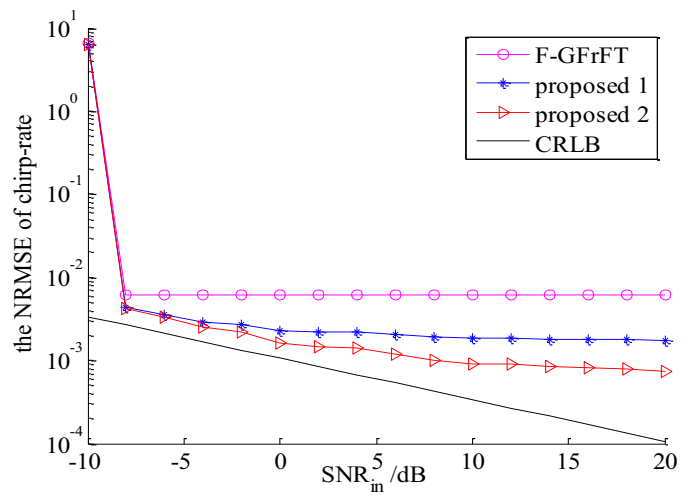

Figure 4. The NRMSE of chirp-rate as a function of $\mathrm{SNR}_{\text {in }}$

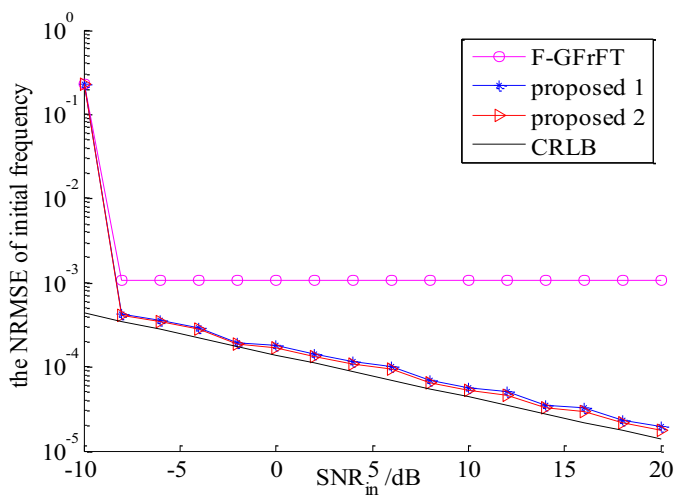

Figure 5. The NRMSE of initial frequency as a function of $\mathrm{SNR}_{\mathrm{in}}$ 
From Figure 4 and Figure 5 we can know that the proposed algorithms have a higher accuracy than the F-GFrFT algorithm both in estimating the chirp-rate and initial frequency, especially the in estimating the initial frequency. The enhancement algorithm has more advantages in the estimation of the chirp-rate while the initial frequency estimation performance is slightly better.

\section{Conclusions and Future Work}

According to the practical circumstances we have met in radar signal processing, a new model is established. Besides, the quadratic interpolation algorithm is proposed via Lagrange interpolation polynomial, and it can be applied to arbitrary windowed LFM signal. We demonstrated through simulations that the new signal processing model is very suitable for parameter estimation under low duty ratio and low SNR environment, and it can improve the SNR when the duty ratio is less than 0.9. And the quadratic interpolation algorithm we have proposed can effectively improve the accuracy of parameter estimation and outperforms the contrast algorithm in terms of NRMSE.

It should be pointed out that the new algorithm approaches the CRLB when estimating the initial frequency, but there still is an obvious gap between the CRLB when estimating the chirp-rate in high SNR. As the future work, we shall analyze the source of the error and further improve the accuracy of the algorithm.

\section{References}

1. V. Namias. The fractional order Fourier transform and its application to quantum mechanics. IMA Journal of Applied Mathematics. 25.3 (1980) 241-265.

2. H.M. Ozaktas, O. Arikan, M.A. Kutay, et al. Digital computation of the fractional Fourier transform. IEEE Transactions on signal processing. 44.9 (1996) 2141-2150.

3. X.H. Zhao, R. Tao, B. Deng, et al. New methods for fast computation of fractional Fourier transform. Dianzi Xuebao(Acta Electronica Sinica). 35.6 (2007) 1089-1093.

4. Y. Ding, L. Sun,H. Zhang, et al. A multi-component LFM signal parameters estimation method using STFT and Zoom-FRFT. Communication Software and Networks (ICCSN), 2016 8th IEEE International Conference on. IEEE. (2016) 112-117.

5. F. Zhang, L. Qi, E. Chen, et al. Parameter estimation of LFM signal in the fractional fourier domain via curve-fitting optimization technique. Pervasive Computing Signal Processing and Applications (PCSPA), 2010 First International Conference on. IEEE. (2010) 582-585

6. J. Song, Y. Liu, X. Zhu. Parameters estimation of LFM signals by interpolation based on FRFT. Systems Engineering and Electronics. 33.10 (2011) 2188-2193.

7. J. Song, Y. Wang, Y. Liu. Iterative interpolation for parameter estimation of LFM signal based on fractional Fourier transform. Circuits, Systems, and Signal Processing. 32.3 (2013) 1489-1499.

8. R. Chen, Y. Wang. Universal FRFT-based algorithm for parameter estimation of chirp signals. Journal of Systems Engineering and Electronics. 23.4 (2012) 495-501.

9. L. J. Stanković, T. Alieva, M. J. Bastiaans. Time-frequency signal analysis based on the windowed fractional Fourier transform. Signal Processing. 83.11 (2003): 2459-2468.

10. P. Wang, T. S. Qiu, et al. Parameters estimation of LFM signal based on Gaussian-weighted fractional Fourier transform. Journal on Communications. 37.4 (2016) 107-115.

11. R. Chen, Y. Wang. Efficient detection of chirp signals based on the fourth-order origin moment of fractional spectrum. Circuits, Systems, and Signal Processing. 33.5 (2014) 1585-1596.

12. B. Ristic, B. Boashash. Comments on" The Cramer-Rao lower bounds for signals with constant amplitude and polynomial phase. IEEE Transactions on Signal Processing. 46.6 (1998) 17081709 .

13. J. C. Liu, et al. SNR analysis of LFM signal with Gaussian white noise in fractional Fourier transform domain. Journal of electronics \& information technology. 29.10 (2007) 2337-2340. 
14. X. W. Jin, M. F. Lu, Y. A. Xie, et al. Sampled signal analysis in the fractional Fourier transform domain. URSI Asia-Pacific Radio Science Conference (URSI AP-RASC). IEEE. (2016) 14891492. 